\title{
Variations in the Levels of Mulberroside A, Oxyresveratrol, and Resveratrol in Mulberries in Different Seasons and during Growth
}

\author{
Jin Zhou, ${ }^{1,2}$ Shun-xiang Li, ${ }^{1,2}$ Wei Wang, ${ }^{1}$ Xiao-yi Guo, ${ }^{1}$ Xiang-yang Lu, ${ }^{2}$ \\ Xin-pei Yan, ${ }^{3}$ Dan Huang, ${ }^{1}$ Bao-yang Wei, ${ }^{2}$ and Liang Cao ${ }^{1}$ \\ ${ }^{1}$ School of Pharmacy, Hunan University of Chinese Medicine, Changsha 410208, China \\ ${ }^{2}$ School of Bioscience \& Biotechnology, Hunan Agricultural University, Changsha 410128, China \\ ${ }^{3}$ Hunan Institute of Sericulture, Changsha 410127, China \\ Correspondence should be addressed to Shun-xiang Li; lishunxiang@hotmail.com \\ and Xiang-yang Lu; xiangyangcn@163.com
}

Received 27 June 2013; Accepted 17 July 2013

Academic Editors: S. E. Harris and T. Kaneta

Copyright (C) 2013 Jin Zhou et al. This is an open access article distributed under the Creative Commons Attribution License, which permits unrestricted use, distribution, and reproduction in any medium, provided the original work is properly cited.

\begin{abstract}
This study aimed to investigate the composition of three major stilbenes (mulberroside A, oxyresveratrol, and resveratrol) in different portions of mulberries collected in different seasons and their change molds during growth by high-performance liquid chromatography. Mulberroside A levels were the highest in the bark and roots of Morus atropurpurea Roxb, Morus alba Linn, and Morus latifolia Poir. Oxyresveratrol levels were the highest in roots and stem. Both of these high levels were in September. The amount of resveratrol was very low in all samples. In the stem, Morus latifolia Poir contained more mulberroside A than the other two mulberries. Mulberroside A was not detected in the leaves of the three mulberries. In Morus atropurpurea Roxb seedlings, the root tended to contain more of the three stilbenes than leaves. The temporal peaks of resveratrol were always ahead of those for oxyresveratrol. The levels of the stilbenes varied in different portions of the varieties of mulberries collected in different season and in the seedlings of Morus atropurpurea Roxb.
\end{abstract}

\section{Introduction}

Stilbenic compounds (mulberroside A, oxyresveratrol, and resveratrol) (Figure 1) have been investigated for a wide range of bioactivities including antitumor properties [1], antityrosinase [2], antiviral [3], neuroprotective [4], antioxidant activities [5], and a higher protective effect on DNA [6]. Almost all products are extracted from mulberry cortex for there is no effective method to synthesize these compounds [7]. In addition, little is known about the composition and change patterns of stilbenes during their growth in mulberries. Therefore, this study aimed to investigate the composition of the three major stilbenes in different portions of mulberries collected in different seasons, as well as their change molds during growth, by high-performance liquid chromatography (HPLC).

\section{Experimental}

2.1. Chemicals and Materials. Acetonitrile was of HPLC grade (Tedia, USA). Methanol and alcohol were of analytical grade from Tianjin Ke-Miou Reagent Company (Tianjin, China). Ultrapure water was purified by Milli-Q system (Millipore, Bedford, MA, USA). Formic acid was of analytical grade from Tianjin Fuyu Reagent Company (Tianjin, China). Mulberroside A (123-100311), oxyresveratrol (2009031102), and resveratrol (111535-200502) were from Tianjin Kuiqing Reagent Company (Tianjin, China), Mingyuan Reagent Company (Tianjin, China), and Meidi Reagent Company (Zhejiang, China) and were all proved to be above $98 \%$ by HPLC analysis. All samples of mulberries were gathered from the Hunan Institute of Sericulture (Changsha, 


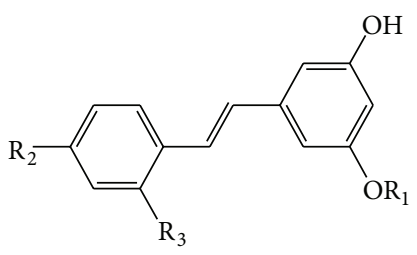

$$
\begin{array}{llll}
1 & \mathrm{R}_{1}=\mathrm{Glu} & \mathrm{R}_{2}=\mathrm{O}-\mathrm{Glu} & \mathrm{R}_{3}=\mathrm{OH} \\
2 & \mathrm{R}_{1}=\mathrm{H} & \mathrm{R}_{2}=\mathrm{OH} & \mathrm{R}_{3}=\mathrm{OH} \\
3 & \mathrm{R}_{1}=\mathrm{H} & \mathrm{R}_{2}=\mathrm{OH} & \mathrm{R}_{3}=\mathrm{H}
\end{array}
$$

FIGURE 1: Structures of compounds 1-3 identified from mulberry. 1 mulberroside A; 2 oxyresveratrol; 3 resveratrol.

China) and authenticated by Professor Yan XP of the Hunan Institute of Sericulture (Changsha, China).

\subsection{Apparatus and Chromatographic Conditions. An Agilent} 1100 liquid chromatography system (Agilent Technologies Deutschland, Waldbronn, Germany), armed with a quaternary solvent delivery system and ultraviolet detector, was used. All analyses were performed with a Hypersil BDS C18 column $(200 \mathrm{~mm} \times 4.6 \mathrm{~mm}, 5 \mu \mathrm{m})$ at a temperature of $40^{\circ} \mathrm{C}$. Ultraviolet absorption was set at $320 \mathrm{~nm}$, and an HS3120 ultrasonic purger was obtained from Jiangsu Hanbon Science \& Technology Company (Jiangsu, China). Eluent A (acetonitrile) and $\mathrm{B}(1.0 \%$ aqueous formic acid, $v / v)$ were used with the gradient program set as follows: $0-25 \mathrm{~min}$, linear change from A-B $(5: 95, v / v)$ to A-B $(30: 70, v / v)$. Reequilibration interval was $15 \mathrm{~min}$ between individual runs with the flow rate $1.0 \mathrm{~mL} \mathrm{~min}^{-1}$. The aliquots of $10 \mu \mathrm{L}$ were injected each time.

2.3. Standard Solutions Preparation. Reference compounds 1-3 (1-mulberroside A, 2-oxyresveratrol, 3-resveratrol) were prepared as follows: following accurately weighed and dissolved in $60 \%$ methanol, the compounds were diluted to the concentration ranges of $\mathbf{1}\left(0.64-404.40 \mu \mathrm{g} \mathrm{mL}^{-1}\right), 2(0.71-$ $\left.444.00 \mu \mathrm{g} \mathrm{mL}^{-1}\right)$, and $\mathbf{3}\left(0.72-448.00 \mu \mathrm{g} \mathrm{mL}^{-1}\right.$ ) (see Table 1 ).

2.4. Sample Preparation. Randomly selected samples of mulberry were first air-dried, milled into powder, dried at room temperature until constant weight, and then passed through a 40-mesh sieve, followed by ultrasonic extraction with $25 \mathrm{~mL}$ (for $0.5 \mathrm{~g}$ ) of $60 \%$ methanol for $40 \mathrm{~min}$. After that, the solvent was again added to the resultant mixture to make it equal to the original weight prior to the ultrasonic extraction, followed by filtering the supernatant through a $0.45 \mu \mathrm{m}$ membrane just before HPLC injection. All samples were prepared in triplicate.

Methods to optimize the extraction conditions, calibration graphs, limit of detection (LOD) and quantification (LOQ), and method of validation and application were adapted from the previously reported systems $[8,9]$. Briefly, the methods were validated as follows.
2.5. Optimization of Extraction Conditions. In the preliminary study, we found that, compared with other methods, ultrasonic extraction was more effective with less interference. Different concentrations of methanol and ethanol were tested for their efficiency as a solvent. As $60 \%$ of methanol as a solvent produces the highest yields for all constituents, it was chosen in the current study. The impact of the length of the extraction time on the efficiency of extraction was evaluated as well. Tested with $60 \%$ methanol for $10,20,40$, and 60 mins, respectively, powdered samples extracted the highest amount of constituents when treated for 40 mins. When column temperature was maintained at $40^{\circ} \mathrm{C}$ instead of 20 or $30^{\circ} \mathrm{C}$, optimized separation was achieved. Various mobile phase compositions were also tested. Results show that water/acetonitrile mixture, not methanol/water mixture, can obtain satisfactory resolution. Addition of acid $(0.5 \%$ formic acid, $0.5 \%$ acetic acid, and $1.0 \%$ formic acid) in the mobile phase improves resolution and reduces the peak tailing of the target compounds, with the best results obtained when using acetonitrile/water mixture with $1.0 \%$ formic acid. The previously mentioned optimized conditions were used in the current study. According to the absorption maxima of three standards on the ultraviolet spectrum, with threedimensional chromatograms of HPLC-DAD detection, the wavelength of $320 \mathrm{~nm}$ was used in the study.

2.6. Calibration Graphs, LODs, and LOQs. The concentration of the compounds was determined by external standard method. Linear regression analyses for each compound were conducted by plotting the peak area versus concentration. The calibration curve for each compound was composed of six points representing six different concentrations in triplicate. The results are shown in Table 1 . All the compounds show linearity $\left(r^{2}>0.9999\right)$ in a relatively wide concentration range.

The LOD and LOQ for each compound under the chromatographic conditions were obtained by measuring the amount of analytical background (Table 1). The signal-tonoise $(\mathrm{S} / \mathrm{N})$ ratio for each compound obtained by injecting a series of solutions is $\mathbf{3}$ for LOD and 10 for LOQ.

2.7. Method Validation. The repeatability of the method was tested by intra- and interday variability. Six replicate samples were extracted and analyzed within one day to determine the intraday variability, and the same sample was used on six independent days to obtain the interday variability. The quantity of each ingredient in the sample was determined from its corresponding calibration curve. The relative standard deviation (RSD) obtained by six replicated injections of the solution was taken as a measure of method repeatability. As shown in Table 2, the intra- and interday RSD values of the three compounds are all less than $2.5 \%$, implying good reproducibility.

The recovery test was done by spiking a solution containing known quantities of the standard and known amounts of powdered mulberry samples, mixed prior to extraction. The standard solutions with their concentration levels in the middle part of the calibration curve and six fortified samples 
TABLE 1: Linear relation between peak area and concentration $(n=6)$.

\begin{tabular}{lccccc}
\hline Compound & Regression equation & $r^{2}$ & Linear range $\left(\mu g \mathrm{~mL}^{-1}\right)$ & $\mathrm{LOD}^{\mathrm{c}}\left(\mathrm{ng} \mathrm{mL}^{-1}\right)$ & $\mathrm{LOQ}^{\mathrm{d}}\left(\mathrm{ng} \mathrm{mL}^{-1}\right)$ \\
\hline $\mathbf{1}$ & $y=22.804 x-25.360$ & 0.9999 & $0.64-404.40$ & 2.60 & 8.67 \\
$\mathbf{2}$ & $y=54.197 x-144.350$ & 0.9999 & $0.71-444.00$ & 2.10 & 7.00 \\
$\mathbf{3}$ & $y=75.172 x-71.439$ & 1 & $0.72-448.00$ & 0.95 & 3.17 \\
\hline
\end{tabular}

${ }^{\mathrm{a}}$ In the regression equation $y=a x+b, x$ refers to the concentration of the compound $\left(\mu \mathrm{g} \mathrm{mL}^{-1}\right), y$ the peak area.

${ }^{\mathrm{b}} r^{2}$ is the correlation coefficient of the equation.

${ }^{\mathrm{c}} \mathrm{LOD}$ : limit of detection.

${ }^{\mathrm{d}}$ LOQ: limit of quantification.

TABLE 2: Intra- and interday repeatability and recovery of the three major stilbenes in mulberry.

\begin{tabular}{|c|c|c|c|c|c|c|}
\hline \multirow[b]{2}{*}{ Compound } & \multicolumn{4}{|c|}{ Repeatability $(n=6)$} & \multicolumn{2}{|c|}{ Recovery $(n=6)$} \\
\hline & $\begin{array}{c}\text { Intraday } \\
\text { Mean } \pm \text { S.D. }\end{array}$ & $\begin{array}{c}\text { R.S.D. } \\
(\%)\end{array}$ & $\begin{array}{c}\text { Interday } \\
\text { Mean } \pm \text { S.D. }\end{array}$ & $\begin{array}{c}\text { R.S.D. } \\
(\%)\end{array}$ & $\begin{array}{l}\text { Mean } \\
(\%)^{\mathrm{b}}\end{array}$ & $\begin{array}{l}\text { R.S.D. } \\
(\%)^{c}\end{array}$ \\
\hline 1 & $1140.2 \pm 12.5$ & 1.1 & $1138.0 \pm 14.8$ & 1.3 & 103.5 & 2.4 \\
\hline 2 & $840.3 \pm 9.2$ & 1.1 & $846.4 \pm 14.4$ & 1.7 & 102.4 & 1.9 \\
\hline 3 & $61.2 \pm 0.9$ & 1.5 & $60.8 \pm 1.4$ & 2.3 & 100.0 & 2.3 \\
\hline
\end{tabular}

${ }^{\mathrm{a}}$ Data were $\mu \mathrm{g}$ constituents per gram drug.

${ }^{\mathrm{b}}$ Calculated as detected amount/added amount $\times 100 \%$. Data were means of six experiments.

${ }^{c}$ R.S.D. $(\%)=($ S.D. $/$ mean $) \times 100$.

were applied. The recovery rate of the method was 100.0$103.5 \%$, with RSD less than $2.5 \%$, suggesting that the method is accurate (Table 2).

Stability of the solutions of the samples was tested by comparing sample solutions that were kept at room temperature with the standard solutions every $2 \mathrm{~h}$ within $24 \mathrm{~h}$, and we found that the sample solutions were stable within $12 \mathrm{~h}$ (RSD $<1.7 \%$ ).

\section{Results and Discussion}

All three stilbenes in mulberries were evaluated by a developed analytical method as described in what follows. Peaks in the achieved chromatograms were recognized by comparing the retention times and ultraviolet spectra with those of standard solutions. Representative chromatograms are shown in Figure 2. Retention parameters for $\mathbf{1 - 3}$ were 7.65, 16.33, and $20.15 \mathrm{~min}$, respectively.

The complete summary of the results of mulberroside A, oxyresveratrol, and resveratrol from different portions of Morus atropurpurea Roxb, Morus alba Linn, and Morus latifolia Poir collected in different seasons is shown in Tables 3 and 4 and that from seedlings of Morus atropurpurea Roxb is shown in Table 5.

In this study, we found that mulberroside $\mathrm{A}$ was richest in bark and roots in September, oxyresveratrol was richest in roots and stem in September as well, and resveratrol was very low in all. Mulberroside A levels were the highest in the bark and roots of Morus atropurpurea Roxb, Morus alba Linn and Morus latifolia Poir. In the stem, Morus latifolia Poir contained the highest level of mulberroside A, but it was undetected in the leaves of Morus atropurpurea Roxb, Morus alba Linn, and Morus latifolia Poir.

Although they have been found in mulberry wood [10], the amount and the relative quantity of oxyresveratrol and resveratrol in mulberry bark, pith, roots, or tuber were uncertain. In the current study, we found that many parts of mulberry contained more oxyresveratrol than resveratrol (Tables 3 and 4), and in general, the levels of both are much less than mulberroside A. September is the best time to obtain both oxyresveratrol and resveratrol. It seems that the parts and varieties of mulberry, as well as season, are all factors influencing the levels of mulberroside A, oxyresveratrol, and resveratrol.

Glycosylation of polyphenolic compounds is a common feature in plants, which can enhance the stability of compounds [11]. In the case of glycosylation of stilbenes, it may protect them from oxidation and enzymatic degradation and thus enhances their stability. In the processes of glycosylation of stilbenes, free stilbene is first synthesized and then glycosylated by endogenous glycosyltransferases [12].

The major role of stilbenes in a number of plant families, such as peanut, mulberry, and grapevine, is working as phytoalexins [13], a group of low-weight molecules with protective functions produced by plants in response to infection [14]. In this context, our finding of a higher level of stilbenes in tubers than in other parts of Morus atropurpurea Roxb (Table 4) is expected.

The formation of stilbene phytoalexins involves the phenylalanine/polymalonate route (Figure 3), and the key step in this biosynthesis pathway is catalyzed by stilbene synthase (STS), which exerts iterative condensation reactions with malonyl-CoA $[12,15,16]$. With the starter coenzyme A-esters of cinnamic acid derivatives ( $\mathrm{p}$-coumaroyl-CoA in the case of resveratrol or cinnamoyl-CoA in the case of pinosylvin) and three malonyl-CoA units, STS can produce the stilbene phytoalexins in one reaction. Leaves which were suggested to be the site of stilbene biosynthesis as two peaks of STS mRNAs in grapevine leaves treated by ultraviolet light were observed by Douillet-Breuil et al. [17]. In the current study, stilbenes were found to be richest in roots, suggesting that there may be another site for stilbene biosynthesis. 


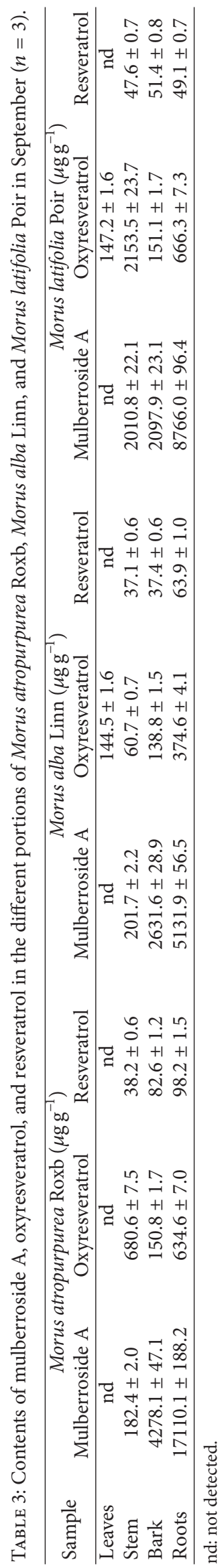




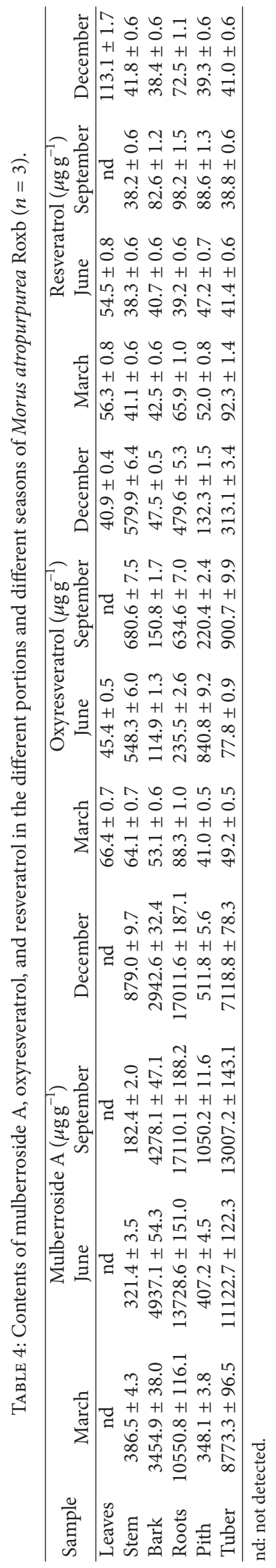




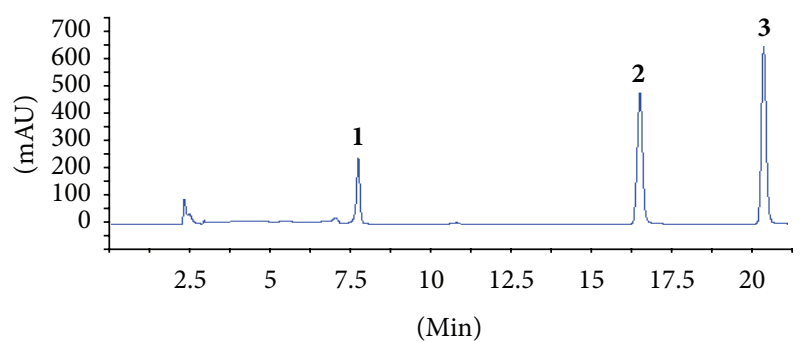

(a)

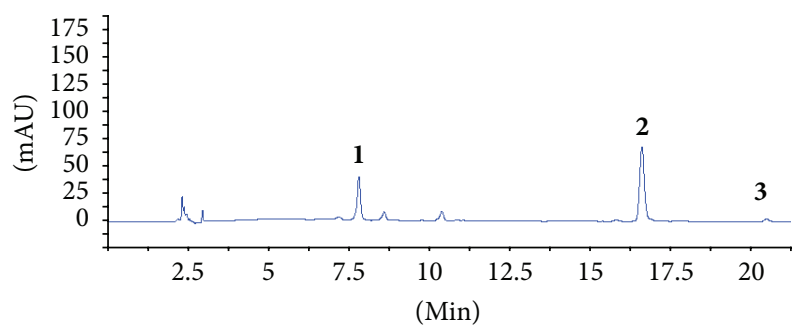

(c)

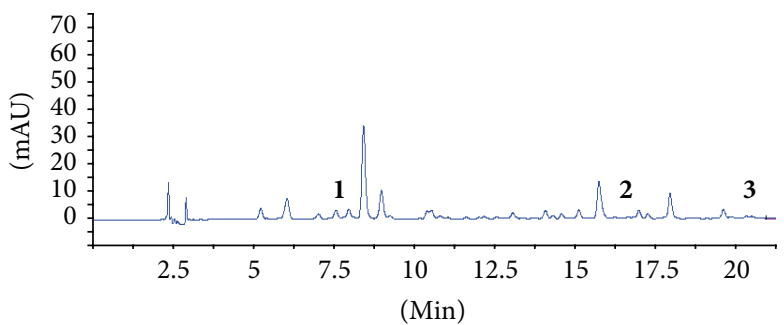

(b)

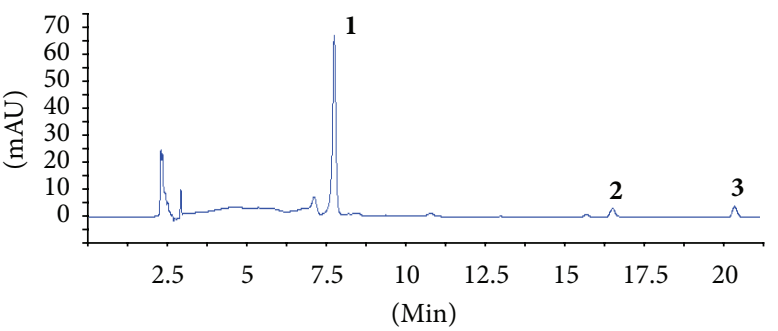

(d)

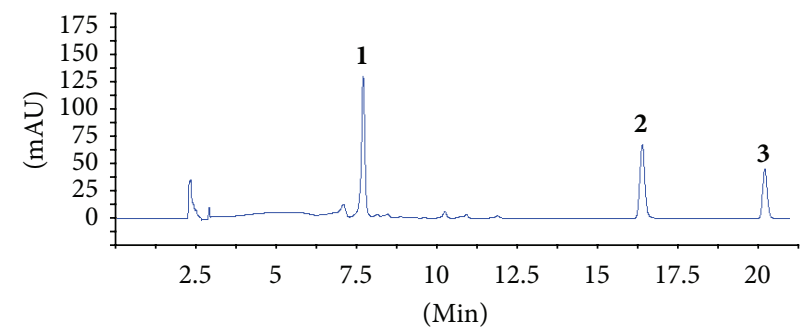

(e)

FIgURE 2: HPLC chromatograms of standard mixture (a), leaves of Morus atropurpurea Roxb (b), stem of Morus atropurpurea Roxb (c), bark of Morus atropurpurea Roxb (d), and roots of Morus atropurpurea Roxb (e). 1 mulberroside A; 2 oxyresveratrol; 3 resveratrol.

TABLE 5: Contents of components of Morus atropurpurea Roxb seedling $(n=3)$.

\begin{tabular}{lccc}
\hline Sample & $\begin{array}{c}\text { Mulberroside A } \\
\left(\mu \mathrm{gg}^{-1}\right)\end{array}$ & $\begin{array}{c}\text { Oxyresveratrol } \\
\left(\mu \mathrm{g} \mathrm{g}^{-1}\right)\end{array}$ & $\begin{array}{c}\text { Resveratrol } \\
\left(\mu \mathrm{gg}^{-1}\right)\end{array}$ \\
\hline Leaves (7th day) & nd & $39.0 \pm 0.4$ & $37.5 \pm 0.6$ \\
Leaves (9th day) & nd & $47.2 \pm 0.5$ & $226.8 \pm 3.4$ \\
Leaves (11st day) & nd & $51.1 \pm 0.6$ & $44.2 \pm 0.7$ \\
Leaves (13rd day) & nd & $69.4 \pm 0.8$ & $76.0 \pm 1.1$ \\
Leaves (15th day) & nd & $70.8 \pm 0.8$ & $46.2 \pm 0.7$ \\
Leaves (17th day) & nd & $114.2 \pm 1.3$ & $91.7 \pm 1.4$ \\
Leaves (20th day) & nd & $67.0 \pm 0.7$ & $42.4 \pm 0.6$ \\
Roots (7th day) & $354.2 \pm 3.9$ & $51.7 \pm 0.6$ & $220.2 \pm 3.3$ \\
Roots (9th day) & $362.4 \pm 4.0$ & $69.1 \pm 0.8$ & $175.9 \pm 2.6$ \\
Roots (11st day) & $413.8 \pm 4.6$ & $71.9 \pm 0.8$ & $108.1 \pm 1.6$ \\
Roots (13rd day) & $1128.1 \pm 12.4$ & $82.4 \pm 0.9$ & $211.2 \pm 3.2$ \\
Roots (15th day) & $923.7 \pm 10.2$ & $129.1 \pm 1.4$ & $111.4 \pm 1.7$ \\
Roots (17th day) & $631.0 \pm 6.9$ & $193.8 \pm 2.1$ & $73.1 \pm 1.1$ \\
Roots (20th day) & $1015.2 \pm 11.2$ & $55.9 \pm 0.6$ & $43.6 \pm 0.7$ \\
\hline
\end{tabular}

nd: not detected.

In order to get new insight about stilbene biotransformation, we examined three major stilbenes in leaves and roots of seedlings of mulberry. This is the first time, to the best of our knowledge, that the amount of mulberroside A, oxyresveratrol, and resveratrol in Morus atropurpurea Roxb seedling leaves and roots has been quantified (Table 5). Among Morus atropurpurea Roxb seedlings of the 7th day20th day, mulberroside A was not detected in the leaves, while it varied between 354.2 and $1128.1 \mu \mathrm{g} \mathrm{g}^{-1}$ in the roots and reached its peaks on the 13 th day and in 20 th day, respectively. Oxyresveratrol peaked on the 17 th day with $114.2 \mu \mathrm{gg}^{-1}$ in leaves and $193.8 \mathrm{\mu g} \mathrm{g}^{-1}$ in roots. In leaves, on the 9th day, the level of resveratrol was $226.8 \mu \mathrm{g} \mathrm{g}^{-1}$, while oxyresveratrol was only $47.2 \mu \mathrm{gg}^{-1}$. Resveratrol has two peaks in roots, $220.2 \mu \mathrm{gg}^{-1}$ on the 7 th day and $211.2 \mu \mathrm{gg}^{-1}$ on the 13th day. As in both leaves and roots, the peaks for resveratrol always run ahead of oxyresveratrol in time, it is logical to propose that oxyresveratrol is probably transformed from resveratrol through oxidation.

\section{Conclusions}

The levels of the stilbenes vary in different parts of varieties of mulberries collected in different seasons and in the seedlings of Morus atropurpurea Roxb. The method has been proved to be simple, rapid, and accurate and can be readily used to determine the content of the major stilbenes in mulberries. 


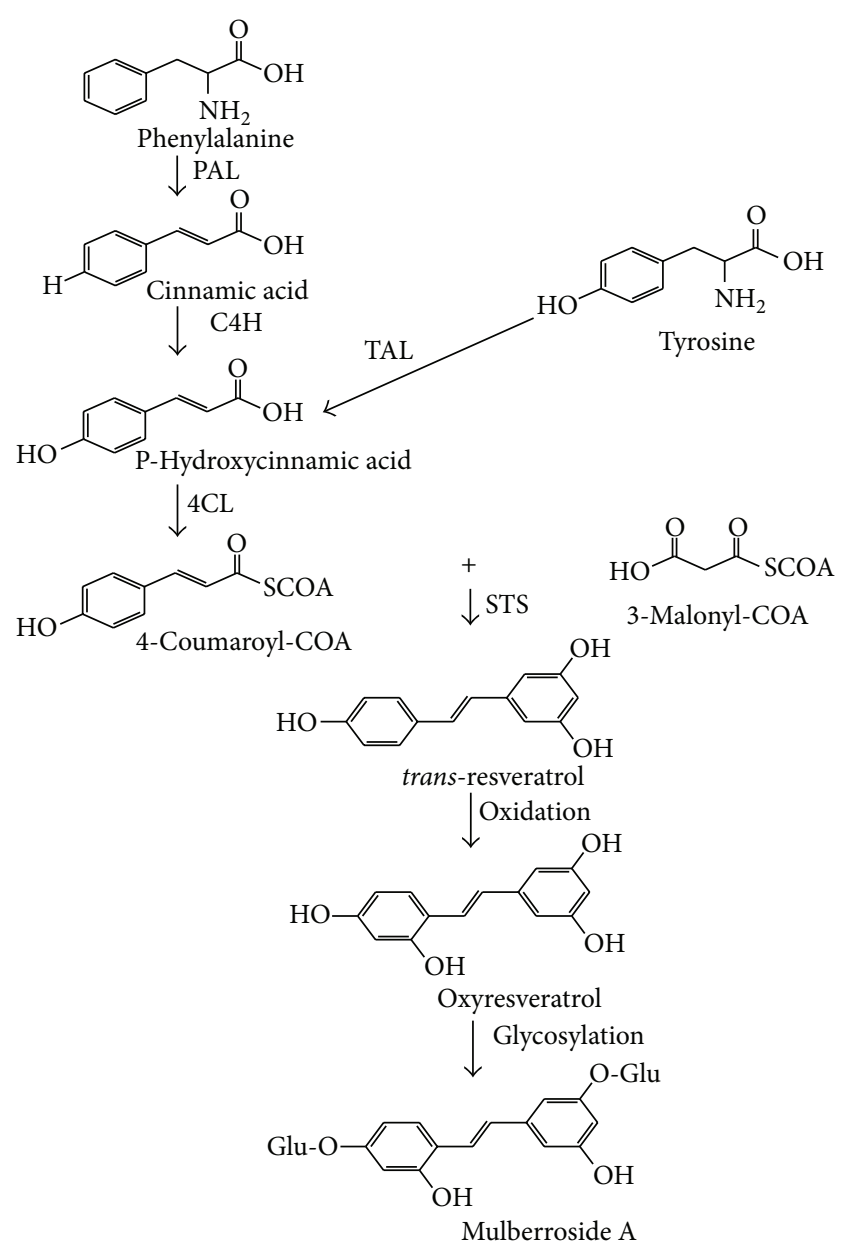

Figure 3: Biosynthesis of resveratrol via the phenylalanine/polymalonate pathway. Phenylalanine ammonia lyase (PAL); tyrosine ammonia lyase (TAL); cinnamate-4-hydroxylase (C4H); 4-coenzyme A ligase (4CL); stilbene synthase (STS). Adapted from Jeandet et al. [12].

\section{Acknowledgments}

The authors thank the National Key Technology R\&D Program (2012BAD36B07), Hunan Provincial S\&T Project (2010FJ1010-3, 2012FJ4291), the project of 12th-five of Hunan Province in Key Disciplines of Pharmacy (2011) no. 76, and the Research Program of the Education Department of Hunan Province (10A091) for financial support of this work. The authors sincerely thank Professor RAO LQ. (School of Bioscience \& Biotechnology, Hunan Agricultural University) for his kind help.

\section{References}

[1] R. Chillemi, S. Sciuto, C. Spatafora, and C. Tringali, "Anti-tumor properties of stilbene-based resveratrol analogues: recent results," Natural Product Communications, vol. 2, pp. 499-513, 2007.

[2] P. Tengamnuay, K. Pengrungruangwong, I. Pheansri, and K. Likhitwitayawuid, "Artocarpus lakoocha heartwood extract as a novel cosmetic ingredient: evaluation of the in vitro antityrosinase and in vivo skin whitening activities," International Journal of Cosmetic Science, vol. 28, no. 4, pp. 269-276, 2006.

[3] P. Sasivimolphan, V. Lipipun, K. Likhitwitayawuid et al., "Inhibitory activity of oxyresveratrol on wild-type and drug-resistant varicella-zoster virus replication in vitro," Antiviral Research, vol. 84, no. 1, pp. 95-97, 2009.

[4] S. A. Andrabi, M. G. Spina, P. Lorenz, U. Ebmeyer, G. Wolf, and T. F. W. Horn, "Oxyresveratrol (trans-2,3',4,5' -tetrahydroxystilbene) is neuroprotective and inhibits the apoptotic cell death in transient cerebral ischemia," Brain Research, vol. 1017, no. 1-2, pp. 98-107, 2004.

[5] P. Lorenz, S. Roychowdhury, M. Engelmann, G. Wolf, and T. F. W. Horn, "Oxyresveratrol and resveratrol are potent antioxidants and free radical scavengers: effect on nitrosative and oxidative stress derived from microglial cells," Nitric Oxide, vol. 9, no. 2, pp. 64-76, 2003.

[6] M. Chatsumpun, T. Chuanasa, B. Sritularak, and K. Likhitwitayawuid, "Oxyresveratrol protects against DNA damage induced by photosensitized riboflavin," Natural Product Communications, vol. 6, no. 1, pp. 41-44, 2011.

[7] E. Alonso, D. J. Ramón, and M. Yus, "Simple synthesis of 5substituted resorcinols: a revisited family of interesting bioactive molecules," Journal of Organic Chemistry, vol. 62, no. 2, pp. 417-421, 1997.

[8] B. Shao, H.-Z. Guo, Y.-J. Cui et al., "Simultaneous determination of six major stilbenes and flavonoids in Smilax china by high performance liquid chromatography," Journal of Pharmaceutical and Biomedical Analysis, vol. 44, no. 3, pp. 737-742, 2007.

[9] H. Huang, M. Liang, P. Jiang, Y. Li, W. Zhang, and Q. Gong, "Quality evaluation of Rhodiola crenulata: quantitative and qualitative analysis of ten main components by HPLC," Journal of Liquid Chromatography and Related Technologies, vol. 31, no. 9, pp. 1324-1336, 2008.

[10] Y. M. Kim, J. Yun, C.-K. Lee, H. Lee, K. R. Min, and Y. Kim, "Oxyresveratrol and hydroxystilbene compounds. Inhibitory effect on tyrosinase and mechanism of action," Journal of Biological Chemistry, vol. 277, no. 18, pp. 16340-16344, 2002.

[11] J. Chong, A. Poutaraud, and P. Hugueney, "Metabolism and roles of stilbenes in plants," Plant Science, vol. 177, no. 3, pp. 143$155,2009$.

[12] P. Jeandet, B. Delaunois, A. Conreux et al., "Biosynthesis, metabolism, molecular engineering, and biological functions of stilbene phytoalexins in plants," BioFactors, vol. 36, no. 5, pp. 331-341, 2010.

[13] P. Langcake and R. J. Pryce, "A new class of phytoalexins from grapevines," Experientia, vol. 33, no. 2, pp. 151-152, 1977.

[14] K. O. Müller and H. Börger, "Experimentelle Untersuchungen über die Phytophthora- Resistenz der Kartoffel," Arbeiten aus der Biologischen Reichsanstalt für Land-und Forstwirtschaft, vol. 23, pp. 189-231, 1940.

[15] M. B. Austin and J. P. Noel, "The chalcone synthase superfamily of type III polyketide synthases," Natural Product Reports, vol. 20, no. 1, pp. 79-110, 2003.

[16] O. Yu and J. M. Jez, "Nature's assembly line: biosynthesis of simple phenylpropanoids and polyketides," Plant Journal, vol. 54, no. 4, pp. 750-762, 2008.

[17] A.-C. Douillet-Breuil, P. Jeandet, M. Adrian, and R. Bessis, "Changes in the phytoalexin content of various Vitis spp. in response to ultraviolet C elicitation," Journal of Agricultural and Food Chemistry, vol. 47, no. 10, pp. 4456-4461, 1999. 

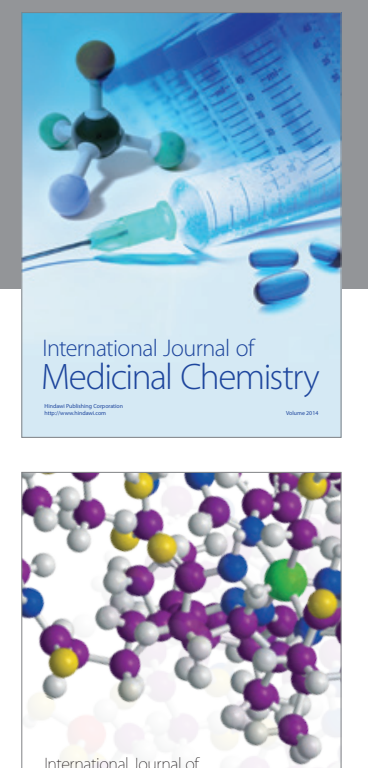

\section{Carbohydrate} Chemistry

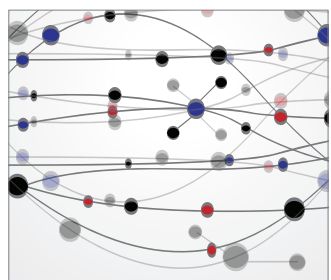

The Scientific World Journal
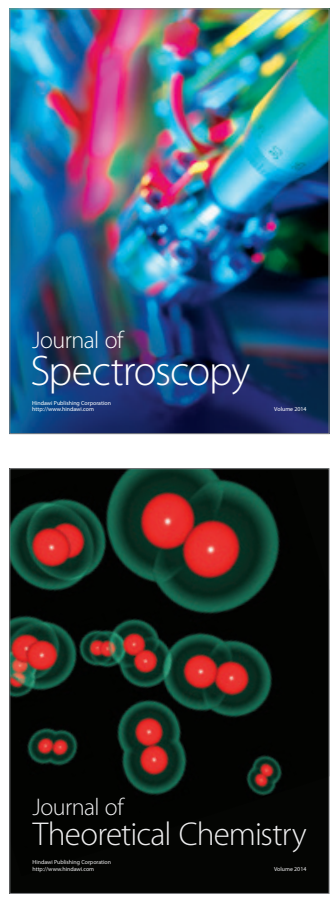
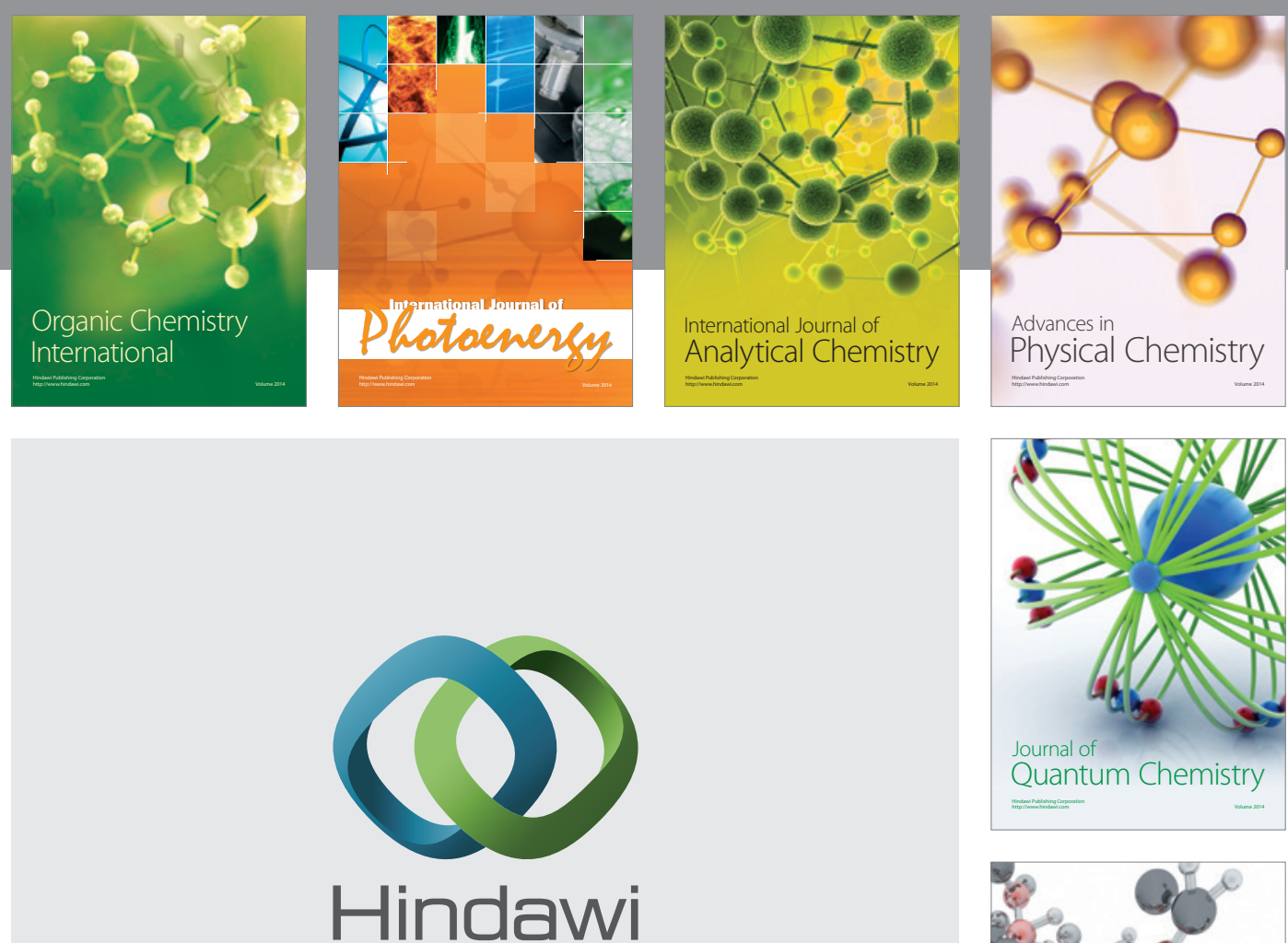

Submit your manuscripts at

http://www.hindawi.com

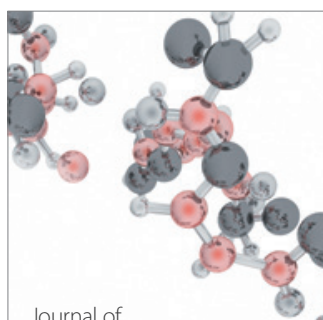

Analytical Methods

in Chemistry

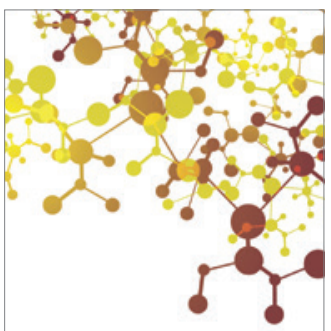

Journal of

Applied Chemistry

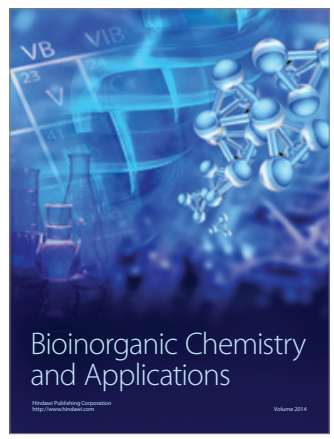

Inorganic Chemistry
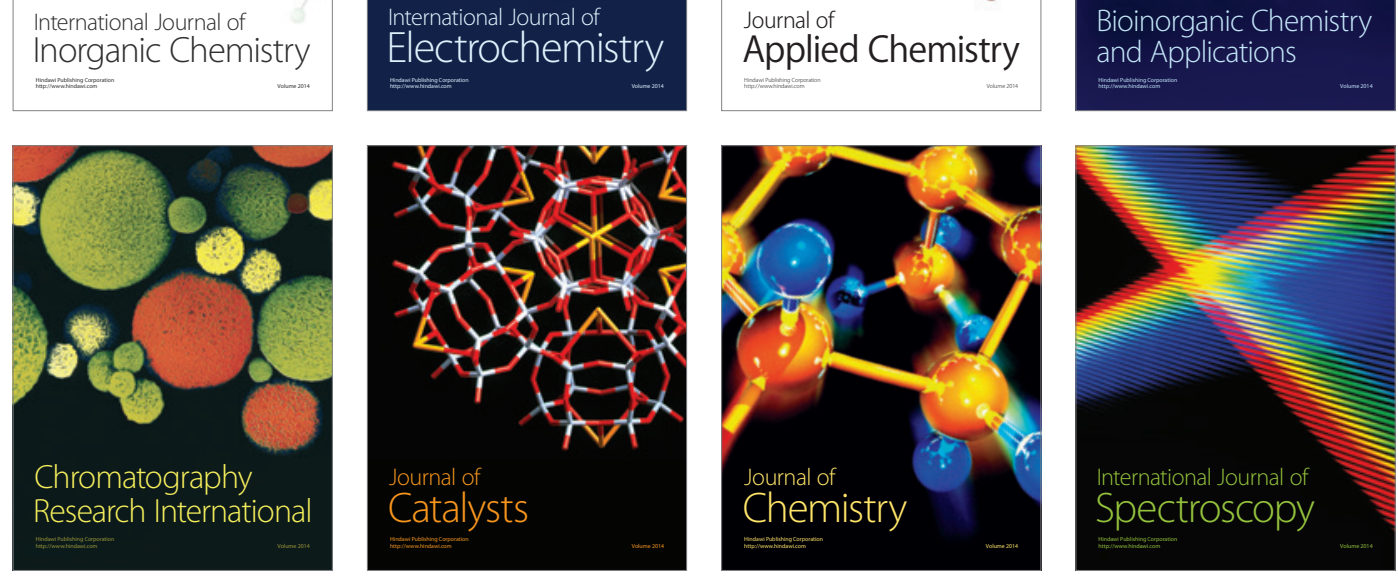